\title{
Comparison of the Effects of Dobutamine and Milrinone on Hemodynamic Parameters and Oxygen Supply in Patients Undergoing Cardiac Surgery with Low Cardiac Output after Anesthetic Induction
}

\author{
Maria José Carvalho Carmona, TSA ${ }^{1}$, Laura Mariana Martins ${ }^{2}$, Matheus Fachini Vane ${ }^{2}$, Breno Altero Longo ${ }^{2}$, \\ Lemuel Silva Paredes ${ }^{3}$, Luiz Marcelo Sá Malbouisson, TSA ${ }^{4}$
}

\begin{abstract}
Summary: Carmona MJC, Martins LM, Vane MF, Longo BA, Paredes LS, Malbouisson LMS - Comparison of the Effects of Dobutamine and Milrinone on Hemodynamic Parameters and Oxygen Supply in Patients Undergoing Cardiac Surgery with Low Cardiac Output after Anesthetic Induction.

Background and objectives: Several classes of inotropic drugs with different hemodynamic effects are used in the treatment of low cardiac output in patients with diastolic dysfunction undergoing cardiac surgery. The objective of the present study was to compare the effects of dobutamine and milrinone on hemodynamic parameters and oxygen supply in this population of patients.
\end{abstract}

\begin{abstract}
Methods: After approval by the Ethics Committee of the institution and signing of the informed consent, 20 patients undergoing cardiac surgery with cardiac index $<2 \mathrm{~L} \cdot \mathrm{min}^{-1} \cdot \mathrm{m}^{2}$ after anesthetic induction and placement of a pulmonary artery catheter were randomly divided to receive dobutamine $5 \mu \mathrm{g} \cdot \mathrm{kg}^{-1}$. $\min ^{-1}(n=10)$, or milrinone $0.5 \mu \mathrm{g} \cdot \mathrm{kg}^{-1} \cdot \mathrm{min}^{-1}(n=10)$. Hemodynamic parameters were measured after anesthetic induction and after 30 and 60 minutes, and arterial and venous blood gases were measured at baseline and 60 minutes. Non-paired Student $t$ test or two-way ANOVA for repeated measurements was used to compare the data.
\end{abstract}

Results: Dobutamine and milrinone promoted significant increases in cardiac index (56\% and $47 \%$ ) and oxygen supply (53\% and $45 \%$ ), and reduction in systemic (33\% and $36 \%$ ) and pulmonary (34\% and 19\%) vascular resistance, respectively. However, statistically significant differences were not observed between both drugs.

Conclusions: Both inotropic drugs were similarly effective in restoring tissue blood flow and oxygen supply to adequate levels in patients with low cardiac output undergoing cardiac surgery.

Keywords: ANESTHESIA, General; COMPLICATIONS: Iow cardiac output; DRUGS, Vasodilators: dobutamine, milrinone; SURGERY, Cardiac: myocardial revascularization.

\section{INTRODUCTION}

Ventricular dysfunction can be seen, in different perioperative periods, in patients undergoing cardiac surgery, with the consequent inadequate oxygen supply to body tissues. After adjusting the blood volume, hemodynamic support with varying doses of inotropic drugs and/or vasodilators can contribute for hemodynamic adequacy. When the need of inotropic support in situations of low cardiac output is identified, dobutamine is one of the drugs

Received from the Discipline of Anesthesiology of the Faculdade de Medicina da Universidade de São Paulo (FMUSP), SP

1. Full Professor Associated to the Discipline of Anesthesiology of the FMUSP; Director of the Division of Anesthesiology of the Instituto Central do Hospital das Clínicas of the FMUSP

2. Undergraduate student of the FMUSP

3. Assistant physician of the Service of Anesthesiology of the Instituto do Coração of the Hospital das Clínicas of the FMUSP

4. Doctor in Sciences; Coordinator-Physician of the ICU of the Discipline of Anesthesiology of the FMUSP

Submitted on April 2, 2009

Approved on January 20, 2010

Correspondence to:

Dra. Maria José Carvalho Carmona

Divisão de Anestesia do ICHC

Av. Enéas Carvalho de Aguiar, 255, 80 andar - Cerqueira César

05403-900 - São Paulo, SP, Brasil

E-mail: maria.carmona@incor.usp.br used more often. More recently, phosphodiesterase inhibitors like milrinone have been indicated for this purpose ${ }^{1}$.

In a study comparing the effects of milrinone and dobutamine in patients with low cardiac output after myocardial revascularization, the efficacy of both drugs was observed. However, patients who received dobutamine had a higher increase in cardiac output, heart rate, and mean arterial pressure while in the milrinone group patients had greater reduction in pulmonary wedge pressure ${ }^{2}$. As for side effects observed in this study, dobutamine was associated with a higher number of cases of increased blood pressure and atrial fibrillation, while the incidence of sinus bradycardia was higher in patients treated with milrinone. This greater reduction in pulmonary wedge pressure related to milrinone can contribute for faster reduction in pulmonary congestion in patients with congestive heart failure ${ }^{2,3}$. However, in other studies with patients with heart failure, significant differences in parameters related to right ventricular function, mortality, need of other vasodilator or inotropic drug, or need of mechanical circulatory support in cardiac transplantation with the isolate use of one of those two drugs were not observed ${ }^{4,5}$.

Therefore, a consensus on the appropriate selection of inotropic therapy in ventricular dysfunction does not exist, and it 
is up to the physician to evaluate the hemodynamic status of the patient and choose the most adequate vasoactive drug at that moment, or for a drug association.

Severe preoperative ventricular dysfunction is one of the indications for monitoring with the pulmonary artery catheter in patients undergoing cardiac surgery. When low cardiac output is identified immediately after anesthetic induction, besides correction of the blood volume and administration of drugs that could potentially depress the myocardium, the use of inotropic or vasodilator drugs such as dobutamine and/or milrinone can contribute for the adjustment of oxygen supply to the tissues, preventing organ dysfunctions that could compromise the postoperative evolution of patients.

The objective of the present study was to compare the effects of dobutamine and milrinone on hemodynamics and oxygen supply in patients undergoing cardiac surgery who developed low cardiac output non-responsive to volume adjustment after anesthetic induction.

\section{METHODS}

After approval by the Research Ethics Commission of the Hospital das Clínicas da Faculdade de Medicina da Universidade de São Paulo, 20 patients undergoing cardiac surgery under general anesthesia with minimal or moderate risk according to the evaluation proposed by Higgins et al. ${ }^{6}$, and who developed low cardiac output (cardiac index lower than 2.0 L. min $^{-1}$. $\mathrm{m}^{2}$ ) immediately after anesthetic induction and hemodynamic monitoring, without adequate response to blood volume adjustment, with pulmonary artery pressure $>18 \mathrm{mmHg}$, were included in this study.

Patients with indication for the use of vasoactive and/or vasodilators to maintain hemodynamic stability before anesthetic induction or distribution to participate in the study were excluded.

Oral midazolam, 0.1 to $0.2 \mathrm{mg} \cdot \mathrm{kg}^{-1}$ up to $15 \mathrm{mg}$, was administered 30 minutes before the surgery. After admission to the operating room, patients were monitored with continuous electrocardiogram on the $D_{\|}$derivation, pulse oximeter, and invasive blood pressure obtained by inserting a pressure transducer in the radial artery. After pre-oxygenation and induction of general anesthesia, patients were ventilated manually with a facemask and intubated with an ET tube with the proper diameter, following by the onset of controlled mechanical ventilation. Proper positioning of the tracheal tube was determined by bilateral pulmonary auscultation. After anesthetic induction, hemodynamic parameters were monitored by a 7.5F Swan-Ganz catheter (pulmonary artery catheter, Baxter Critical Care, California, USA) introduced through the right internal jugular vein. During anesthetic induction each patient received $500 \mathrm{~mL}$ of Ringer's lactate to maintain a peripheral venous access and infusion of drugs.

After the proper positioning of the Swan-Ganz catheter in the pulmonary artery, the blood volume was adjusted at the discretion of the anesthesiologist, and hemodynamic parameters, such as heart rate, blood pressure, mean pulmonary artery pressure, central venous pressure, pulmonary wedge pressure, cardiac output, cardiac index, systemic vascular resistance index, pulmonary vascular resistance index, left ventricular work index, and right ventricular work index were evaluated. Cardiac output was determined by intermittent thermodilution, with three different measurements and using the mean of those measurements. Blood samples were drawn for hemoglobin levels, hematocrit, and arterial blood gases. With the results of the hemodynamic parameters and laboratorial exams, the relationship among the arterial oxygen pressure and inspired oxygen fraction, alveolararterial gradient of oxygen $\left(\mathrm{AAO}_{2} \mathrm{G}\right)$, pulmonary shunt, arteriovenous oxygen difference $\left(\mathrm{DAVO}_{2}\right)$, oxygen consumption index $\left(\mathrm{IVO}_{2}\right)$, oxygen delivery index $\left(\mathrm{IDO}_{2}\right)$, and oxygen extraction $\left(\mathrm{EO}_{2}\right)$ were calculated.

The inclusion criterion was the presence of a cardiac index lower than 2.0 L. $\mathrm{min}^{-1} \cdot \mathrm{m}^{2}$ at the time of the evaluation of hemodynamic parameters and basal oxygen supply in the absence of vasoactive drugs. Patients were randomly divided in two groups, according to the inotropic agent administered:

- Group D - dobutamine, $5 \mu \mathrm{g} \cdot \mathrm{kg}^{-1} \cdot \mathrm{min}^{-1}$

- Group $\mathrm{M}-$ milrinone, $0.5 \mu \mathrm{g} \cdot \mathrm{kg}^{-1} \cdot \mathrm{min}^{-1}$

After beginning the infusion of one of the drugs through the atrial port of the Swan-Ganz catheter, hemodynamic parameters were evaluated in 30 and 60 minutes, and blood was draw at 60 minutes, at the end of the protocol, to calculate oxygen supply. When cardiopulmonary bypass (CPB) was initiated before the end of the study, the case was excluded.

The score proposed by Higgins et al. ${ }^{6}$ was calculated to evaluate the surgical risk of patients undergoing myocardial revascularization. Classification of the surgical risk of patients followed the criteria in Table I.

Non-paired Student $t$ test was used to compare demographic data, surgical risk, and laboratorial exams. Two-way

Table I - Surgical Risk Classification

\begin{tabular}{ll}
\hline Criteria & Points \\
\hline Emergency surgery & 6 \\
Serum creatinine > 1.9 & 4 \\
Serum creatinine between 1.4 -1.9 & 1 \\
LVEF < 50\% & 3 \\
Repeated cardiac surgery & 3 \\
Presence of mitral insufficiency & 3 \\
Age above 75 years & 2 \\
Age between 64 and 75 years & 1 \\
Prior vascular surgery & 2 \\
Diagnosis of Chronic Obstructive Pulmonary Disease & 2 \\
Hematocrit lower than 34\% & 2 \\
Presence of aortic stenosis & 1 \\
Diabetes under treatment & 1 \\
Presence of coagulopathies & 1 \\
Diagnosis of cerebral vascular disease & 1 \\
Weight lower than 65 kg & 1 \\
\hline
\end{tabular}

LVEF - left ventricular ejection fraction

Score evaluation: $0-1$ points: minimal risk; $2-3$ points: low risk; $4-6$ points: moderate risk; $7-9$ points: high risk; > 10 points: extremely risky. 
ANOVA for repeated measures was used to compare hemodynamic parameters and oxygen supply, and the drug administered to the patient was considered the intergroup factor and blood drawings as repeated measurements. In case of differences between groups or moments, or the presence of interaction, multiple comparisons for the significant factor were carried out with the Student-Newman-Keuls test. A $p<$ 0.05 was considered significant.

\section{RESULTS}

Table II shows the data regarding gender, weight, height, BMI, and surgical risk. Demographic characteristics, surgical risk, and preoperative laboratorial evaluation were similar in both groups.

After induction of anesthesia, the hemodynamic performance of patients in both groups, characterized by low cardiac indices (Group D = $1.59 \pm 0.20$ and Group M = $1.44 \pm 0.38$ L. min $^{-1}$. $\mathrm{m}^{2}$ ), elevated systemic (Group $\mathrm{D}=2577 \pm 630$ and Group $M=$ $3107 \pm 1008$ dyn. $\mathrm{s}^{-1} \cdot \mathrm{cm}^{-5} \cdot \mathrm{m}^{-2}$ ) and pulmonary (Group D $=469 \pm$ 184 and Group $M=507 \pm 259$ dyn. $\mathrm{s}^{-1} \cdot \mathrm{cm}^{-5} \cdot \mathrm{m}^{-2}$ ) vascular resistance, and low left ventricular (Group D = 29 \pm 10 and Group $\mathrm{M}=22 \pm 8 \mathrm{~kg} \cdot \mathrm{min}^{-1} \cdot \mathrm{m}^{-2}$ ) and right ventricular (Group D = $12 \pm$ 12 and Group $M=10 \pm 4 \mathrm{~kg} \cdot \mathrm{min}^{-1} \cdot \mathrm{m}^{-2}$ ) work indices, was considered inadequate. Central venous pressure (Group D = $17 \pm 4$ and Group $\mathrm{M}=15 \pm 4 \mathrm{mmHg}$ ) and pulmonary wedge pressure (Group D = $22 \pm 5$ and Group $\mathrm{M}=22 \pm 7 \mathrm{mmHg}$ ) were elevated. Oxygen supply and consumption were low, and oxygen extraction was elevated in both groups greater than $30 \%$. Statistically significant differences in basal hemodynamic parameters were not observed in both groups. It was not necessary to administer volume to all patients, since pulmonary wedge pressure was greater than $18 \mathrm{mmHg}$, before the administration of the chosen vasoactive drug.

As shown in Table III the introduction of vasoactive drugs promoted significant and sustained increase in the cardiac in-

Table II - Demographic Data and Surgical Risk of Patients in the Dobutamine and Milrinone Groups

\begin{tabular}{llll}
\hline & Dobutamina & Milrinone & p value \\
\cline { 2 - 4 } Gender (M/F; $)$ & $(5 / 5)$ & $(6 / 4)$ & NS \\
Age (years) & $69 \pm 8$ & $62 \pm 10$ & NS \\
BMI (kg.cm-2) & $28.4 \pm 3.8$ & 26.13 .5 & NS \\
Weight (kg) & $75 \pm 14$ & $68 \pm 11$ & NS \\
Height (cm) & $164 \pm 10$ & $162 \pm 7$ & NS \\
Surgical risk & $4.4 \pm 1.4$ & $4.1 \pm 1.8$ & NS \\
LVEF (\%) & $47 \pm 15$ & $43 \pm 22$ & NS \\
Creatinine (mg.dL-1) & $1.2 \pm 0.2$ & $1.4 \pm 0.4$ &
\end{tabular}

$\mathrm{BMI}=$ body mass index; $\mathrm{LVEF}=$ left ventricular ejection fraction.

Table III - Hemodynamic Parameters During the Study

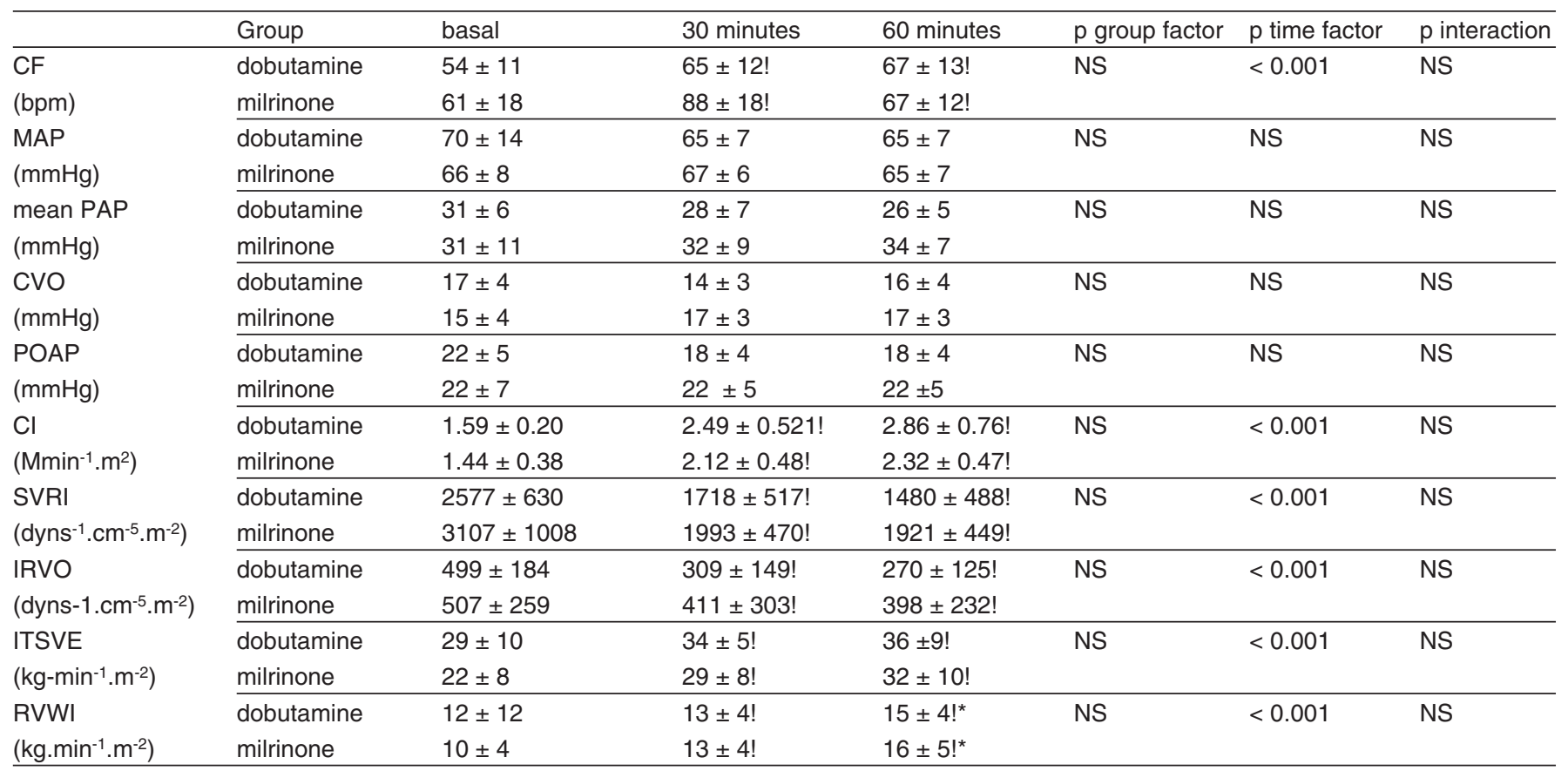

$\mathrm{HR}=$ heart rate; MAP = mean arterial pressure; mean PAP = mean pulmonary artery pressure; CVP = central venous pressure; PCWP = pulmonary capillary wedge pressure; $\mathrm{Cl}=$ cardiac index; SVRI = systemic vascular resistance index; PVRI = pulmonary vascular resistance index; $\mathrm{LVWI}=$ left ventricular work index; RVWI = right ventricular work index. ! means $p<0.05$ when compared to baseline. * means $p<0.05$ when compared with levels at 30 minutes. 
Table IV - Oxygenation and Oxygen Supply Parameters

\begin{tabular}{|c|c|c|c|c|c|c|}
\hline & Grupo & basal & 60 minutos & $\mathrm{p}$ group factor & $\mathrm{p}$ time factor & $\mathrm{p}$ interaction \\
\hline \multirow[t]{2}{*}{$\mathrm{PaO}_{2} / \mathrm{FiO}_{2}$} & dobutamine & $438 \pm 191$ & $307 \pm 148$ & NS & $<0.001$ & NS \\
\hline & milrinone & $411 \pm 78$ & $298 \pm 119$ & & & \\
\hline $\mathrm{GAaO}_{2}$ & milrinone & $127 \pm 52$ & $211 \pm 117$ & & & \\
\hline \multirow[t]{2}{*}{$\mathrm{DavO}_{2}(\mathrm{mmHg})$} & dobutamine & $5.5 \pm 1.5$ & $3.8 \pm 1$ & NS & $<0.001$ & NS \\
\hline & milrinone & $6.5 \pm 1.6$ & $4.5 \pm 1.5$ & & & \\
\hline $\mathrm{IVO}_{2}\left(\mathrm{mlmin}^{-1} \cdot \mathrm{m}^{-2}\right)$ & milrinone & $97 \pm 34$ & $100 \pm 18$ & & & \\
\hline \multirow[t]{2}{*}{$\mathrm{IDO}_{2}\left(\mathrm{mlmin}^{-1} \cdot \mathrm{m}^{-2}\right)$} & dobutamine & $279 \pm 60$ & $429 \pm 135$ & NS & $<0.001$ & NS \\
\hline & milrinone & $263 \pm 72$ & $381 \pm 81$ & & & \\
\hline \multirow[t]{2}{*}{$\mathrm{EO}_{2}(\%)$} & dobutamine & $32 \pm 9$ & $26 \pm 8$ & NS & $<0.006$ & NS \\
\hline & milrinone & $36 \pm 7$ & $27 \pm 8$ & & & \\
\hline
\end{tabular}

$\mathrm{PaO}_{2} / \mathrm{FiO}_{2}=$ partial pressure of oxygen /inspired fraction of $\mathrm{O}_{2} ; \mathrm{AAO}_{2} \mathrm{G}=$ alveolar-arterial oxygen gradient; DAVO ${ }_{2}=$ arteriovenous oxygen difference; IVO $\mathrm{I}_{2}=$ oxygen consumption index; $\mathrm{IDO}_{2}=$ oxygen delivery index; $\mathrm{EO}_{2}=$ oxygen extraction .

dex of $56 \%$ in the Dobutamine Group, and $47 \%$ in the Milrinone Group without statistically significant differences between both groups. The heart rate increased by $20 \%$ with dobutamine, and $11 \%$ with milrinone, and remained elevated until the end of the study without statistically significant differences between both groups. Right and left ventricular work index had similar increases in both groups, while the reduction in systemic and pulmonary vascular resistance was significant and similar in both groups.

When blood oxygenation and oxygen supply were evaluated, a reduction in $\mathrm{PaO}_{2} / \mathrm{FiO}_{2}$ was observed 60 minutes after the onset of the vasoactive drugs, without statistically significant differences between milrinone and dobutamine, which can be seen in Table IV. However, an increase in oxygen supply and reduction in oxygen extraction was observed 60 minutes after the onset of the infusion of vasoactive drugs. Changes in oxygen consumption were not observed.

\section{DISCUSSION}

The main findings of this study were: 1) both inotropic drugs were effective in increasing the cardiac output due to the mild increase in heart rate and systolic work of both ventricles; 2) oxygen supply increased significantly while peripheral oxygen extraction decreased; and 3) significant changes were not observed in oxygen consumption.

Very few studies in the literature compared the isolated action of those two drugs. In prior studies that compared both drugs in the treatment of patients with heart failure, significant differences in right cardiac hemodynamic parameters, mortality, need of another vasodilator or inotropic drug, or the need of mechanical cardiac support after the transplant, were not observed between both drugs 4,5 .

Different hemodynamic effects have been proposed to suggest possible indications for the use of different inotropic drugs. In experimental studies, it was observed that milrino- ne produced greater reduction in total peripheral vascular resistance, as well as in renal and splanchnic resistance when compared to dobutamine, probably resulting from direct vasodilation ${ }^{7}$. This observation was independent from the inotropic effect of the drug. Studying the impact of dobutamine and milrinone on right ventricular function in patients with congestive heart failure, Eichhorn et al. observed that doses of both drugs used to promote similar elevation in cardiac index and left ventricular systolic performance caused different effects on pulmonary circulation. The authors observed that milrinone promoted significant reduction in pulmonary artery pressure and pulmonary vascular resistance, which was not seen with dobutamine ${ }^{8}$.

In different clinical contexts, significant differences in the hemodynamic impact of those drugs were not observed. According to Feneck et al., who compared both drugs in patients with low cardiac output after myocardial revascularization, both drugs were adequate and effective in the treatment of low cardiac output, but a higher increase in $\mathrm{CI}, \mathrm{HR}, \mathrm{MAP}$, and LVWI was observed with dobutamine, while the Milrinone Group showed greater reduction in PCWP 2. Comparing the effects of dobutamine and milrinone in patients awaiting heart transplant, Aranda et al. did not observe differences in MPAP, PCWP, pulmonary vascular resistance, BP, HR, and cardiac output ${ }^{4}$.

In a study that evaluated or compared the hemodynamic impact of dobutamine and milrinone in patients with congestive heart failure treated with carvedilol, Lowes et al. observed that milrinone promoted more significant reduction in mean arterial pressure, PCWP, and MAP than dobutamine 9 . Elevated doses of dobutamine $\left(15-20 \mu \mathrm{g} \cdot \mathrm{kg}^{-1} \cdot \mathrm{min}^{-1}\right)$ were necessary to achieve similar elevation in cardiac output and left ventricular work index as milrinone. In those doses, dobutamine caused significant increase in heart rate, but it did not change PCWP. According to those results, Lowes et al. suggested that the hemodynamic profile of dobutamine is less adequate than that of milrinone in patients with heart 
failure treated with beta-blockers. In the present study, both drugs were effective in restoring the cardiac output, and the superiority of the hemodynamic characteristics of one drug over the other was not observed. However, in this study, both drugs were equally effective in restoring the hemodynamic parameters in patients with low cardiac output after anesthetic induction.

Despite the important and significant increase in cardiac index and parameters of oxygen supply, significant changes in consumption of oxygen were not observed. This may indicate that, in the hemodynamic point of view, although oxygen supply to tissues was low before the administration of inotropic drugs, it was adequate for the consumption at that moment in a patient under general anesthesia. Evaluating the impact of each drug separately, the increase in myocardial contractility could be responsible for myocardial oxygen consumption, worsening a probable cardiac ischemia, which would be, in theory, harmful. The administration of both drugs, in the doses used here, promoted the reduction in peripheral and pulmonary vascular resistance, contributing to reduce myocardial oxygen consumption, compensating for the probable negative impact of using inotropic agents.

The limitations of the present study include the number of patients (only 10 in each group), and the time available to evaluate each drug (longer time for analysis was not possible because patients underwent surgical procedures that required CPB). Patients would have an unfavorable evolution without the use of inotropic drugs during the surgery, which makes it impossible to have a control group using placebo. It is known that the parameters investigated are influenced by the level of anesthesia. In this study, the level of anesthesia was not monitored.

The impact of this study relies in the fact that a rule or consensus on the first choice of inotropic therapy in patients undergoing cardiac surgery and who present low cardiac output after anesthetic induction does not exist, and it is up to the anesthesiologist to indicate the inotropic drug or the best association of vasoactive drugs according to his/her experience. Since marked differences were not observed between both treatments, the cost of each medication could be addressed since it is higher for milrinone. 